\title{
The impact of CHP planning restrictions on the efficiency of urban energy systems
}

\author{
James Keirstead*, Nouri Samsatli*, Nilay Shah*, Céline Weber* \\ Department of Chemical Engineering \\ Imperial College London, UK, SW7 2AZ
}

\begin{abstract}
Cities account for approximately two-thirds of global primary energy consumption and have large heat and power demands. Combined heat and power (CHP) systems offer significant primary energy efficiency gains and emissions reductions, but they can have high upfront investment costs and create nuisance pollution within the urban environment. Urban planners therefore need to understand the tradeoffs between limitations on CHP plant size and the performance of the overall energy system. This paper uses a mixed-integer linear programming model to evaluate urban energy system designs for a range of city sizes and technology scenarios. The results suggest that the most cost-effective and energy-efficient scenarios require a mix of technology scales including CHP plants of appropriate size for the total urban demand. For the cities studied here (less than 200000 people), planning restrictions that prevent the use of CHP technologies could lead to total system cost penalties of $2 \%$ (but with significantly different cost structures) and energy-efficiency penalties of up to $24 \%$ when measured against a boiler-only business-as-usual case.
\end{abstract}

Keywords: Optimization, Urban energy systems, Combined heat and power

\footnotetext{
*Corresponding author. Tel: +44 (0)20 7594 6227, Fax: +44 (0)20 75946606.

Email addresses: j.keirstead@imperial.ac.uk (James Keirstead),

n.samsatli@imperial.ac.uk (Nouri Samsatli), n.shah@imperial.ac.uk (Nilay Shah), c.weber@imperial.ac.uk (Céline Weber)
} 


\section{Introduction}

Recent research has shown that cities are responsible for approximately twothirds of global primary energy consumption and $71 \%$ of energy-related direct greenhouse gas emissions [1]. As a result, there is a growing interest in improving the energy efficiency of cities so that environmental impacts are reduced while maintaining economic opportunities and quality of life.

One of the most significant areas for improvement is the integration of energy services such as heat and power. Exergy analyses of cities such as Vienna and London indicate that only about $10-20 \%$ of a fuel's available work is recovered, with significant amounts of exergy lost in the conversion of fossil fuels to electricity or low-temperature heat $[2,3]$. Cogeneration technologies offer the potential to reduce these losses by converting more of the input exergy into useful service. Furthermore, by embedding such systems within the urban fabric, transmission and distribution losses can be significantly reduced.

However the operation of combined heat and power (CHP) technologies within an urban area can be constrained by several factors. Planning restrictions on noise or air quality, for example, might require that such facilities are located on the outskirts of an urban area or restrict the size of feasible technologies [4]. Similarly, space limitations may exclude the use of large systems within dense urban neighbourhoods. Since large CHP plants can attain higher thermodynamic efficiencies and achieve economies of scale in capital costs, limits on the size of urban CHP plants might therefore restrict the overall efficiency of the urban energy system, potentially making it difficult to reach desired energyefficiency and carbon-reduction targets.

The goal of this paper is to understand how such restrictions might quantitatively affect the performance of urban energy systems for the provision of heat and power. To perform this analysis, we first present an optimization model for the strategic design of integrated urban energy systems. Then, using an idealised grid city, we determine the optimal energy supply system for a range of demand patterns and technology mixes. The paper concludes with a summary 
of the main findings, the implications for planners and energy utilities, and a discussion of how the work might be extended.

\section{The technology urban resource network (TURN) model}

This section describes the optimization model used in this paper. The model seeks to identify the combination of urban energy conversion technologies and distribution networks that best meets a given pattern of resource demands varying in time and space. It is therefore called the technology urban resource network (TURN) model.

\subsection{Previous work}

Researchers have been developing models of energy systems for several decades but advances in computing power since the mid-1990s have led to increased variety in both model design and application. A recent review groups these models into seven generic types including simulation, scenario assessment, equilibrium, top-down, bottom-up, operational optimization, and investment optimization [5]. Some specific examples include:

- MARKAL (Market Allocation) and its related models have been used extensively in a wide variety of countries. Its purpose is to consider scenarios for the evolution of a specific energy system, which may be at a variety of scales from district to national, over a period of up to 50 years. It is based on an economic equilibrium framework and consequently is frequently used for national energy policy planning [e.g. 6].

- Decco is a tool for optimizing energy supply systems [7, 8]. Based on a previous modelling framework called NEMESS [9], it uses optimization to determine the relative utilisation of the (pre-specified) available energy technologies at each time interval that minimises some criterion (e.g. primary energy consumption, cost, emissions). The temporal resolution of the model is very high, by default considering one year using intervals of 
one hour, but this is achieved by optimizing (using linear programming) the supply of energy for each interval independently of the others.

- MODEST is an optimization model that aims to minimise investment and operating costs while satisfying energy demands $[10,11]$. It can be used for a wide variety of settings, from local to national level, for a variety of sectors and at various temporal resolutions. The linear-programming formulation used by MODEST ensures that large problems can be handled with relative ease but a disadvantage with this formulation is that the capital investment costs are assumed to be a linear function of installed capacity (i.e. the solution may involve the installation of equipment with unrealistically low capacities). Other limitations are that energy storage is not considered and there is no spatial dependence.

- EnerGIS [12] combines a geographical information system (GIS) with an MILP formulation in order to design district heating and cooling networks. Annual demands for heating, hot water, cooling and electricity are taken from building analysis of the GIS data. These are then used to calculate heating and cooling loads for each type of building based on the outdoor temperature. The network model is quite sophisticated: the supply and return temperatures are calculated in order to ensure that all demands are satisfied at the correct temperatures. The MILP formulation determines the existence of a network in each zone and also whether or not zones are connected (and can therefore transfer energy). The model considers a number of technologies, including combined-cycle gas turbine CHP and heat pumps (the performance of which is dependent on the temperature of the demand and the natural heat source, which is stored in the GIS) but it is restricted to heating and cooling networks.

- A wide body of research is available on building CHP design. One recent example [13] uses an MILP formulation to determine the optimal configuration and operation of a trigeneration system that minimises annualised cost and satisfies the demands for space and water heating, cooling and 
power. Integer variables are used to determine the number of each device present, which may include cogeneration modules, boilers, absorption chillers, vapour-compression chillers and storage for hot and cold utilities. The time horizon is divided into a number of typical days, each of which has 24 one-hour intervals. Since the model was developed for building energy supply, there is no spatial dependence. One weakness in the model is that it only considers a single fuel, so e.g. the benefit of biomass CHP relative to natural gas cannot be explored; neither does it consider renewable energies, such as PV panels and micro-wind turbines.

An interesting alternative approach is presented by [14], who used an analytical approach to optimizing building CHP systems. The methodology identifies the regions where one technology is superior to the other alternatives (e.g. which type of chiller to use to supply cooling). The study found optimal solutions to be those that minimised primary energy consumption; however, other objectives can be easily accommodated. The main drawback with the approach is that it does not consider any time dependence of the demands (and, of course, there is no spatial dependence either) but the authors intend to address this, along with including energy storage, in the future.

Linear programming techniques (both in traditional and mixed-integer formulations) are therefore often used for both investment and operational optimizations. Models can be single- or multi-objective, single- or multi-period, may or may not include a spatial representation of the energy system, and often focus on specific system types such as district heating or biomass $[15,16,17]$. These examples collectively illustrate that model design implicitly requires a trade-off between representational fidelity, model performance, and portability (i.e. use in multiple contexts). 


\subsection{Nomenclature}

\subsubsection{Indices and sets}

$r \quad$ Resource

$i \quad$ Zone

$t \quad$ Time

$p \quad$ Production technology

$\tau \quad$ Transport technology (for resources)

$\sigma \quad$ Storage technology

$\mu \quad$ Metric or key performance indicator

$\mathrm{T}$ Indicates that a contribution to a metric depends on the number of technologies installed

$\mathrm{R}$ Indicates that a contribution to a metric depends on the rate of operation of a technology

$\Sigma_{r} \quad$ Set of storage technologies capable of storing resource $r$

\subsubsection{User-specified parameters}

$\begin{array}{ll}\Delta t_{t} & \text { Duration of time interval t } \\ D_{r i t} & \text { Demand for resource } r \text { in zone } i \text { at time } t \\ \alpha_{r p} & \text { Conversion factor of resource } r \text { in production technology } p \\ l_{i i^{\prime}} & \text { Distance between zones } i \text { and } i^{\prime} \\ \beta_{r \tau}^{\text {src }}, \beta_{r \tau}^{\text {dst }} & \text { Conversion factor for resource } r \text { in transport technology } \tau \text { for } \\ & \text { the source and destination zone respectively } \\ \gamma_{r \tau}^{\text {src }}, \gamma_{r \tau}^{\text {dst }} & \text { Distance-dependent conversion factor for resource } r \text { in transport } \\ & \text { technology } \tau \text { for the source and destination zone respectively } \\ \mathbb{S}_{\sigma}^{\text {max }} & \text { Maximum capacity of storage technology } \sigma \\ w_{\mu} & \text { Weight in the objective function for metric } \mu \\ \mathscr{A} & \text { Capital annualisation factor } \\ c_{\mu \mathrm{R} p}^{\mathscr{P}}, c_{\mu \mathrm{T} p}^{\mathscr{P}} & \text { Impact on metric } \mu \text { of production technology per unit rate } \\ & \text { of operation and per installed technology respectively (similar } \\ & \text { impacts are defined for imports, exports, transport and storage) }\end{array}$




\subsubsection{Continuous variables}

$P_{\text {rit }} \quad$ Net rate of production of resource $r$ in zone $i$ at time $t$

$Q_{\text {rit }} \quad$ Net rate of increase in resource $r$ in zone $i$ at time $t$ due to transport or resources between zones

$I_{\text {rit }} \quad$ Rate of import of resource $r$ in zone $i$ at time $t$

$E_{\text {rit }} \quad$ Rate of export of resource $r$ from zone $i$ at time $t$

$S_{\text {rit }} \quad$ Net rate of storage of resource $r$ from zone $i$ at time $t$

$\mathscr{P}_{\text {pit }} \quad$ Rate of production technology $p$ in zone $i$ at time $t$

$\mathscr{Q}_{\tau i i^{\prime} t} \quad$ Rate of transport technology $\tau$ from zone $i$ to $i^{\prime}$ at time $t$

$C_{\mu}^{I}, C_{\mu}^{E} \quad$ Contribution of imports and exports respectively to performance metric $\mu$

$C_{\mu}^{\mathscr{P}} \quad$ Contribution of all production technologies to performance metric $\mu$

$C_{\mu}^{\mathscr{Q}} \quad$ Contribution of all transport technologies to performance metric $\mu$

$C_{\mu}^{S} \quad$ Contribution of all storage technologies to performance metric $\mu$

$Z \quad$ Objective function

\subsubsection{Integer/binary variables}

$N_{\sigma i}^{S} \quad$ Number of storage facilities of type $\sigma$ in zone $i$

$N_{p i} \quad$ Number of production technologies of type $p$ in zone $i$

\subsection{Model design}

The model presented here seeks to expand on previous work by considering multiple time periods and discrete spatial locations within a single optimization problem that determines the choice of technologies as well as their operation. It is based on the premise that any urban energy system can be represented as a set of resources and a set of technologies that interconvert those resources. Very similar representations have been used to develop batch scheduling formulations in the chemical manufacturing sector, such as the State-Task Network (STN) representation developed by Kondili et al. [18]. The STN represents chemical process recipes using a directed graph comprising two types of node: states and tasks. The states represent a material of a specific state (e.g. composition, temperature and pressure) and the tasks represent chemical processes 
that convert some of the states to other states. Shortly after, Pantelides [19] developed a more general representation, the Resource-Task Network (RTN), in which the resources represented both material states and equipment items; the tasks could be process operations or procedures performed on equipment resources. This approach allows more general scheduling problems to be considered, such as scheduling a multiproduct plant while simultaneously considering sequence-dependent cleaning of the equipment.

Since a representation very close to the STN is used to describe the possible resources and technologies present in an urban energy system, our model is called the Technology Urban Resource Network, or TURN. The concept of urban resources is generalised, in that they can represent energy carriers (such as gas and electricity), non-energy resources (such as potable water) and any other material or energy stream involved in the provision of urban energy services (such as waste heat, carbon dioxide and so on). "Technologies" represent any process that can convert a set of input resources to a set of output resources. For example, a CHP unit might convert a certain amount of natural gas into electricity, high-quality heat, waste heat, carbon dioxide and other atmospheric pollutants. The high-quality heat may then be converted to space and water heat in buildings by using a heat exchanger connected to a district heating network.

The city is divided into a number of zones (of any shape and size), each of which has time-varying resource demands specified by the user. The TURN model will determine how best to satisfy these demands through the provision of technologies in various zones and networks to transport resources between zones. Depending on the problem, this might result in distributed provision of resources with small-scale technologies in each zone; or a large-scale technology in a single zone with a network to transport the resource to the rest of the city; or some combination of these two strategies. The operation of transport processes may also involve other resources, e.g. transporting a liquid fuel by road would require a certain amount of diesel and result in the generation of waste heat, carbon dioxide and other pollutants. Finally, unless the city is 
entirely self-sufficient, it will need to import some resources from other cities and surrounding hinterlands. The model can choose to import any resource into any zone, subject to a number of constraints such as bounds on the rate of import in each zone and imports may be restricted to a certain subset of the zones. Similarly any excess production of resources may be exported from the city.

The main constraint in the TURN model is the resource balance, which is shown in simplified form below:

$$
P_{\text {rit }}+Q_{\text {rit }}+I_{\text {rit }}-E_{\text {rit }}-D_{\text {rit }}=S_{\text {rit }} \quad \forall \text { rit }
$$

where, for resource $r$ in zone $i$ at time $t, P_{\text {rit }}$ is the net production rate, $Q_{r i t}$ is the net inflow of resource from all other zones within the city (transportation), $I_{r i t}$ and $E_{r i t}$ are the rates of import and export across the city boundaries, $D_{\text {rit }}$ is the demand and $S_{r i t}$ is the net rate of surplus production (i.e. the rate at which the resource is stored).

In this aggregated form of the model, one typical day is represented using a small number of time intervals. To avoid complicated storage constraints, the number of intervals is usually limited to one or two. Using only one interval means considering peak demands and not accounting for any storage at all (i.e. $S_{\text {rit }}=0$ ). With two intervals, the energy system can be designed to cope with the peak demands by consuming some stored resources that were produced in surplus during the low-demand periods; in this case, $t \in\{$ peak, average $\}$, for example.

Whereas $I_{r i t}$ and $E_{\text {rit }}$ are degrees of freedom, and $D_{\text {rit }}$ is a parameter (given as input), the other terms are variables that depend on which technologies are selected and their rates of utilization.

$P_{r i t}$ may be positive or negative, representing production or consumption of the resource respectively, and is defined by:

$$
P_{\text {rit }}=\sum_{p} \alpha_{r p} \mathscr{P}_{\text {pit }} \quad \forall \text { rit }
$$


where $\mathscr{P}_{\text {pit }}$ is the total rate of operation of all technologies of type $p$ in zone $i$ at time $t$ and $\alpha_{r p}$ define how much of each resource $r$ is generated per unit rate of operation of technology $p$ (these values are negative for resources that are consumed). $\mathscr{P}_{\text {pit }}$ is constrained by the number of technologies installed (an integer decision variable) and the maximum rate of a single technology.

Similarly, negative $Q_{\text {rit }}$ represents a net outflow of resource to other zones and depends on the rates of all transport technologies connected to zone $i$ :

$$
Q_{r i t}=\sum_{\tau i^{\prime}}\left(\beta_{r \tau}^{\mathrm{src}}+\gamma_{r \tau}^{\mathrm{src}} l_{i i^{\prime}}\right) \mathscr{Q}_{\tau i i^{\prime} t}+\sum_{\tau i^{\prime}}\left(\beta_{r \tau}^{\mathrm{dst}}+\gamma_{r \tau}^{\mathrm{dst}} l_{i^{\prime} i}\right) \mathscr{Q}_{\tau i^{\prime} i t} \quad \forall r i t
$$

where $\mathscr{Q}_{\tau i i^{\prime} t}$ is the rate of operation of transport technology $\tau$ when transporting resource from cell $i$ to $i^{\prime}$ (over a distance $l_{i i^{\prime}}$ ) at time $t$. $\beta$ and $\gamma$ define how much of each resource is consumed or generated in the source and destination cell per unit rate and per unit rate per unit distance respectively: they allow one to define losses and resource requirements for the transport.

Given the rates of operation of the production technologies $\left(\mathscr{P}_{\text {pit }}\right)$ and the transportation technologies $\left(\mathscr{Q}_{\tau i i^{\prime} t}\right)$, and the rates of import and export, a certain amount of net excess of each resource can be produced: $S_{\text {rit }}$. This is a free variable that is subject to two aggregate constraints (somewhat simplified here, for brevity) that limit the amount of resource that can be stored (or consumed from storage). The first is a capacity constraint, based on the total capacity of the installed storage technologies:

$$
\Delta t_{t} S_{r i t} \leq \sum_{\sigma \in \Sigma_{r}} N_{\sigma i}^{S} \mathbb{S}_{\sigma}^{\max } \quad \forall r i t
$$

where $\Delta t_{t}$ is the duration of interval $t, \Sigma_{r}$ is the set of storage technologies $\sigma$ capable of storing resource $r, N_{\sigma i}^{S}$ is the number of storage technologies of type $\sigma$ installed in cell $i$ and $\mathbb{S}_{\sigma}^{\max }$ is the capacity of a single storage technology.

Finally, all surplus production of a resource during one (or more) interval(s) must be balanced exactly by consumption during the others(s), so that there is no net gain at the end of the day: 


$$
\sum_{t} \Delta t_{t} S_{r i t}=0 \quad \forall r i
$$

Note that the above constraint forces $S_{\text {rit }}$ to be zero when there is only one time interval (as in this paper). When there are more than two intervals, equations 4 and 5 do not strictly bound the amount of resource in storage at all times, hence usually no more than two intervals are considered.

The objective function is a weighted sum of performance metrics, $\mu$, (e.g. cost, environmental impact etc.) related to imports and exports of resources and the activities of production, transport and storage technologies:

$$
\min Z=\sum_{\mu} w_{\mu}\left(C_{\mu}^{I}+C_{\mu}^{E}+C_{\mu}^{\mathscr{P}}+C_{\mu}^{\mathscr{Q}}+C_{\mu}^{S}\right) .
$$

The contribution of each type of activity on each metric is defined by the rates of operation and the numbers of technologies installed. For example, the contribution due to production technologies is given by:

$$
C_{\mu}^{\mathscr{P}}=\mathscr{A} \sum_{p i t} c_{\mu \mathrm{R} p}^{\mathscr{P}} \mathscr{P}_{p i t} \Delta t_{t}+\sum_{p i} c_{\mu \mathrm{T} p}^{\mathscr{P}} N_{p i} \quad \forall \mu,
$$

where the first term is the annual impact of operation and the second is the annual impact due to capital expenditure. $\mathscr{A}$ is a constant that annualises the operating activities and $c_{\mu \mathrm{R} p}^{\mathscr{P}}$ is impact on metric $\mu$ per unit rate of operation of technology $p . c_{\mu \mathrm{T} p}^{\mathscr{P} p}$ is the annualised impact on the metric due to purchase of a technology and $N_{p i}$ is the number of technologies purchased.

Since the resource balance allows demands to be met in a variety of ways (e.g. import, local production, or internal transfers), the TURN framework can model complex resource chains, facilitating simultaneous comparisons of diverse energy provision strategies operating at multiple scales. As binary variables represent the locations of technologies and network links, continuous variables represent the rates of processes and all of the constraints are linear, TURN is a mixed-integer linear programming model. A forthcoming manuscript will describe the model in more detail. 


\section{Experimental design}

Previous studies of urban energy systems have often focused on individual cities due to the availability of data or a desire to address specific policy concerns [e.g. 12, 20]. While this is a sensible strategy for many studies, it can limit the applicability of the findings to other contexts since adjusting the model and collecting data for a new city can be time-consuming and difficult. Our tool can use GIS input data to represent existing cities, but the present goal is to identify the broad trends associated with limitations on CHP plant size and so we adopt a generalized model of the city to represent a range of situations.

Each modelled city has an idealised grid layout with individual cells measuring $400 \mathrm{~m} \times 400 \mathrm{~m}$ (16 ha.); connections between cells are allowed in the Moore neighbourhood (eight neighbours). We assume that the housing density within the city varies linearly with the distance from the centre with some random variation added. Using London as a template, the minimum and maximum housing densities were taken to be 10 and 65 dwellings per hectare respectively; the average household size was 2.3 people. 2007 energy consumption data for each London borough was then used to calculate the annual average power demands per capita for natural gas and electricity $(1.1$ and $0.60 \mathrm{~kW}$ per capita respectively) [21]. As the model requires heat demand as the input, it was assumed that the natural gas was used to provide hot water and space heating in $70 \%$ efficient boilers, the 1996 UK stock average [22]. Using this information, heat and power resource demands were generated for grid cities ranging from 16 to 256 cells $(4 \times 4,6 \times 6, \ldots, 16 \times 16)$, representing populations from 10000 to 200000 residents (see Figure 1 for an example).

For each city configuration, the TURN model was run to determine the optimal (lowest cost) mix of supply technologies and distribution networks that satisfy the demand requirements. Table 1 summarizes the technologies available to the model. The size of the three CHP technologies was selected to span the typical CHP applications described by the UK Combined Heat and Power Association; micro-CHP units (less than $50 \mathrm{~kW}$ ) were omitted as we are interested 
in technologies that can be used as part of a district heating system. Additional data necessary to determine the full cost of the energy system is given in Table 2, such as the costs of building the distribution systems and resource imports.

For each modelled city, the same pattern of demand was used but with five different technology suites: boilers (technology 1 only), small CHP (1-3), medium CHP $(1,2,4)$, large CHP $(1,2,5)$, and all technologies (1-5). Gas boilers were included with the CHP scenarios to ensure that the model did not satisfy the resource balance by over-supplying CHP units.

Several other constraints were added to ensure a sensible solution and to improve performance. First, since the resource balance is enforced per cell and not per household, an unconstrained model could "share" the output of a household-scale technology between households within a single cell. For example, it may select 10 boilers to serve 100 households, rather than 100 boilers each running at $10 \%$. A constraint is therefore added to say that, in addition to any district-scale technologies, each cell must contain as many household-scale technologies as households (namely, gas boilers or heat exchangers). A second related constraint ensures that the minimum load factor of each household technology is $10 \%$ and $30 \%$ for the CHP units. Imports of gas and electricity were also restricted to one cell only, located at the bottom-left corner of the grid.

\section{Results}

The 35 scenarios were run $(7$ grid sizes $\times 5$ technology suites $)$ and a preliminary analysis performed. One of the scenarios was genuinely infeasible: the $4 \times 4$ city had demands too small for the inclusion of a single 50 MW CHP unit. All of the other scenarios were feasible, although the quality of the model solutions varied greatly depending on the problem scaling and optimality gap (i.e. the difference in the objective function value between the integer solution and the fully-relaxed LP, expressed as a percentage of the fully-relaxed LP). For all problems, a termination criteria of $10 \%$ and time-out limits of 20 seconds per cell were used for efficient solving (for an example of how these parameters 
affect model performance, see [17]). However as Figure 2 shows the quality of the solution varied depending on the city size and technology suite being used. The approximately $20 \%$ gaps observed in the larger city sizes are a symptom of degeneracy in the model solution. That is, for a similar value of the objective function, multiple solutions are possible representing rotational, translational or reflective transformations of a similar network design and technology placement.

\subsection{Indicative results}

Each model solution defines an energy supply strategy including distribution networks and the location and number of conversion technologies. To give a brief overview of the model's output, this section considers the results of the $8 \times 8$ case study.

Table 3 summarises the technology combinations used in this case. As noted above, the model must provide each household with either a gas boiler or a heat exchanger connected to the district heating system. The results show that heat exchangers are most prominent in the small CHP case, owing to the costs of building and operating a district heat network (i.e. network lengths are shorter when using many small CHP units compared with several larger CHPs, see discussion in Section 4.3. It is assumed that heat distribution within a cell is included within the installation cost.) The structure of the "all technologies" case is similar to the medium CHP scenario, with $74 \%$ of heat demands being serviced by heat exchangers in both cases.

Figure 3 illustrates the resource networks for the "all technologies" scenario. The city is fuelled primarily by gas, imported at the bottom left of the gas network figure. This is then distributed to ten CHP plants. (Note that we have assumed that there is no additional gas demand, e.g. for cooking.) The two larger $10 \mathrm{MW}$ CHP plants provide much of the heat requirements in the central areas whereas in the peripheral areas of lower demand, gas boilers are used along with eight small CHP systems. The electricity generated by these CHP systems is sufficient to meet $90 \%$ of the city's requirements with grid imports filling the balance. None of the network structures were designed for robustness or other 
performance characteristics and these results therefore indicate average flows or the strategic distribution of resources throughout the city.

\subsection{System costs}

Moving beyond this single case, Figure 4 shows overall trends in the total annual cost of the energy system when normalised by total final energy demand (total system cost includes the cost of imported fuels, as well as the annualised capital and operating costs for the conversion technologies and distribution networks). In almost all scenarios, the most cost-efficient energy system requires a combination of technology scales. The figure also shows how the cost effectiveness depends on appropriate technology sizing. Taking the large CHP technology as an example, a clear decreasing trend can be seen as the city size and its associated demands increase to match the capacity of the 50 MW CHP. However the results also depend on the performance of the optimization model. For example, the results for the 256 cell case are very similar for all technology combinations and, referring to Figure 2, this can be partly explained by the large and variable optimality gap in these solutions. This suggests that 256 cells may represent the useful limit of the model's performance as it is currently configured, although a two-step solution, i.e. where one first aggregates the 256 cells into a coarser 16 cell problem before solving, or other heuristics could be useful for solving problems of this large size.

Total system costs are similar regardless of the size of technology used (inner $90 \%$ quantile $=0.183 \pm 0.015 \mathrm{GBP} /$ delivered kJ). However as shown in Figure 5, the relative share of each cost component does vary by technology. The boiler scenarios are the least capital intensive with equipment costs representing $20 \%$ of the total cost, whereas in the large CHP scenarios, equipment costs represented $53 \%$ of the total costs. The "all technologies" scenarios balance these endpoints with equipment costs constituting $40 \%$ of the total cost. Overall, the "all technologies" scenario is the most cost efficient, saving $1.1 \%$ versus the boiler-only case on average, and the large CHP scenario incurs an average cost premium of $19 \%$ per delivered kilojoule. 


\subsection{System efficiency}

An alternative way of comparing the solutions is to examine their energy efficiency: namely, how much primary energy is required to service the final demands for heat and electricity? For these calculations, we have assumed that imported electricity is produced at $38.7 \%$ efficiency [23] accounting for generation, transmission and distribution losses upstream.

Figure 6 shows that the technology scenarios have unique energy efficiency profiles depending on the size of the city. The efficiency of the boiler-only scenarios for example is constant across all sizes, where as the small and medium CHP cases, as well as the "all technologies" scenario, fluctuate from city to city. In the large CHP case however, the overall system efficiency drops by approximately $20 \%$ as the city gets larger. This is because the distribution losses in the district heat network (powered by a single CHP unit) increase, encouraging the model to use boilers instead of heat exchangers in many dwellings. This can be seen by examining the total length of district heat network constructed for each scenario (Figure 7). The consequences of planning restrictions on CHP technologies therefore depend on the size of the city and the specific technology in question. On average however, the CHP scenarios are 12\% (small), 23\% (medium), and $24 \%$ (large) more energy efficient than the boiler-only case; the "all technologies" scenario is on average $17 \%$ more efficient.

Cost and energy efficiency are considered together in Figure 8. Although this assessment could be handled with a full multi-objective optimization, this basic analysis suggests that the medium CHP solutions represent the best result in most cases (i.e. a balance of high efficiency and low cost). Where the optimizer has found a good quality solution (e.g. all scenarios except the large $14 \times 14$ and $20 \times 20$ cases), the "all technology" solution achieves a similar minimum annual cost, although in the $8 \times 8$ and larger cities the energy-efficiency performance is worse. The exact composition of the "all technology" solution will depend on the specific city in question, but in those studied here, total power demands range from 3 to $61 \mathrm{MW}$ which are similar to the medium CHP's $10 \mathrm{MW}_{e}$ rated capacity, hence the comparability of these results. 
It should be noted that an additional case study was performed to investigate whether the choice of discrete CHP capacities significantly affected the results. We ran the $8 \times 8$ "all technologies" scenario again with a greater selection of CHP technologies at 1, 5, 10, 20 and $50 \mathrm{MW}_{e}$. However the overall solution was structurally similar to the "all technologies" case shown in Table 3 ; that is, eight $1 \mathrm{MW}_{e}$ and two $10 \mathrm{MW}_{e} \mathrm{CHP}$ units with a commensurate mix of heat exchangers and boilers.

\section{Discussion and conclusions}

The goal of this paper was to assess how planning restrictions on the size of combined heat and power systems might impact the overall efficiency of an urban energy system. Using a mixed-integer linear programming model, we simulated seven grid cities of 10000 to 200000 people under five technology scenarios. The results indicate that the most efficient urban energy systems, from both a cost and primary energy-efficiency perspective, require a mix of technologies in order to satisfy demand at an appropriate scale. In the present study, these compromise solutions included a mix of both small and mediumsized CHP systems, as well as gas boilers to serve areas of lower demand density.

The actual penalty incurred by forbidding a particular technology will of course depend on the specific city and its energy service demands. However the results presented here suggest that, by restricting a completely free choice of CHP technologies, the main impact is on energy efficiency. Relative to a business-as-usual boiler only case, average primary energy savings of up to $24 \%$ were observed with the use of CHP units. These results vary with city scale, and for larger cities, the losses incurred by using a single large CHP unit instead of multiple smaller units can be a significant factor. In contrast, the impact of a decision to restrict CHP sizes on overall system cost is relatively minor. The total system costs were very similar for the cases studied here although the balance of capital and running costs varies. Decisions to restrict the use of CHP therefore tend to push costs toward the end users, who end up paying for 
individual gas boilers for heat and imported electricity supplies. From a wholesystem perspective, CHP scenarios are more energy efficient and offer lower running costs, but have high capital costs which may be discouraging to some planners and energy utilities. Market structure is therefore key to determining which system outcome is likely to occur. An energy service company for example may be able to benefit from the economies of scale in CHP plants if it has a long-term relationship with consumers.

A related issue is the scale of technologies chosen at the demand side, i.e. within the household. In this study, we assumed that households each needed to own a single boiler or heat exchanger to meet their local demands. However in an earlier study, it was shown that higher efficiencies can be achieved by "sharing" the output of larger household-scale technologies [24]. That is, instead of using individual $15 \mathrm{~kW}$ boilers or heat exchangers, there may be a benefit in adopting $\sim 100 \mathrm{~kW}$ units whose output can be shared among multiple dwellings (e.g. in a block of flats or small neighbourhood). System planners therefore need to evaluate trade-offs at the demand side as well as the supply side, although these meso-scale technologies may require more sophisticated control and institutional arrangements for effective use.

The analysis has highlighted several issues that need to be considered when seeking to improve and generalise the results. First, the cost analysis showed that the structure of a local energy utility, i.e. who pays the upfront costs of equipment versus the running fuel costs, is likely to have a significant impact on which technology mix might be selected. The results presented here were calculated using a whole-system optimization tool which amalgamates individual, corporate and public sector investment decisions. Different results would be expected if the objective function reflected the investment priorities of only one stakeholder. Secondly, cross-scale analyses (i.e. looking at cities of many different sizes) appear to hide many of the economies of scale that one might expect to see from a "larger" city (i.e. more demand in the similar sized city). This suggests that for a given city size, energy systems are guided by unit marginal costs. However across cities, one must consider the significant plant require- 
ments needed to satisfy a large increase in demand (i.e. the average marginal cost) (for more on this distinction, see [25]). Finally a weakness of the present linear formulation is that technology efficiency was assumed to be constant with output. A non-linear formulation, or a piecewise approximation, would therefore help to capture the variations in fuel consumption that occur under part loads, although this additional complexity could result in slower model performance. Similarly the model solutions for many of the cases presented here had large optimality gaps $(\sim 20 \%)$ and these could be improved in practice if developers know which specific sites within the city are candidates for CHP plants.

The paper also alluded to local air pollution as a driver of CHP plant size restrictions. This has not been explicitly considered here, but it clearly offers a profitable area for further research, for example, by adding a monetary penalty to the objective function based on health impacts. Similarly the optimization model could be enhanced to consider network robustness and multi-scale aggregation to improve model performance. Finally a multi-objective optimization framework would be useful for a formal analysis of the cost and energy-efficiency trade-offs highlighted here.

\section{Acknowledgments}

The authors would like to acknowledge the support of BP through the BP Urban Energy Systems project at Imperial College London.

\section{References}

[1] IEA, World Energy Outlook, International Energy Agency, Paris, 2008.

[2] A. Grubler, The Urban Sustainability Challenge, in: Global Carbon Project - Urban and Regional Carbon Management (Ed.), International Symposium Realizing Low Carbon Cities: Bridging Science and Policy, GCPURCM, Nagoya, 2009.

URL http://www.gcp-urcm.org/files/A20090216/2_Grubler.pdf 
[3] F. DeMartial, Exergy analysis of London and sensitivity analysis of an urban layout model, Master's thesis, École des Ponts ParisTech/Imperial College, London (2009).

[4] X. Yuan, J. Kellett, C. Ma, Conflicts between state planning, market development and environmental sustainability: A case study of the electric power industry in Shandong Province, International Journal of Sustainable Development \& World Ecology 15 (5) (2008) 430-439.

[5] D. Connolly, H. Lund, B. Mathiesen, M. Leahy, A review of computer tools for analysing the integration of renewable energy into various energy systems, Applied Energy 87 (4) (2010) 1059-1082.

[6] BERR, The UK MARKAL Energy Model in the 2007 Energy White Paper (2007).

URL http://www.berr.gov.uk/files/file38979.pdf

[7] T. Bruckner, H. Groscurth, R. Kümmel, Competition and synergy between energy technologies in municipal energy systems, Energy 22 (10) (1997) $1005-1014$.

[8] T. Bruckner, R. Morrison, C. Handley, M. Patterson, High-Resolution Modeling of Energy-Services Supply Systems Using decco: Overview and Application to Policy Development, Annals of Operations Research 121 (2003) 151-180.

[9] H. Groscurth, T. Bruckner, R. Kümmel, Modeling of energy-services supply systems, Energy 20 (9) (1995) 941-958.

[10] D. Henning, MODEST: An energy-system optimisation model applicable to local utilities and countries, Energy 22 (12) (1997) 1135-1150.

[11] D. Henning, S. Amiri, K. Holmgren, Modelling and optimisation of electricity, steam and district heating production for a local Swedish utility, European Journal of Operational Research 175 (2) (2006) 1224-1247. 
[12] L. Girardin, F. Marechal, M. Dubuis, N. Calame-Darbellay, D. Favrat, Energis: A geographical information based system for the evaluation of integrated engery conversion systems in urban areas., Energy 35 (2) (2010) $830-840$.

[13] M. A. Lozano, J. C. Ramos, L. M. Serra, Cost optimization of the design of chcp (combined heat, cooling and power) systems under legal constraints, Energy 35 (2) (2010) 794-805.

[14] A. Akisawa, T. Miyazaki, T. Kashiwagi, Theoretical analysis of the optimal configuration of co-generation systems and competitiveness of heating/cooling technologies, Energy 35 (10) (2010) 4071-4078.

[15] H. Sugihara, J. Komoto, K. Tsuji, A multi-objective optimization model for determining urban energy systems under integrated energy service in a specific area, Electrical Engineering in Japan 147 (3) (2004) 20-31.

[16] H. Ren, W. Gao, A MILP model for integrated plan and evaluation of distributed energy systems, Applied Energy 87 (3) (2010) 1001-1014.

[17] S. van Dyken, B. H. Bakken, H. I. Skjelbred, Linear mixed-integer models for biomass supply chains with transport, storage and processing, Energy 35 (3) (2010) 1338-1350.

[18] E. Kondili, C. C. Pantelides, R. W. H. Sargent, A general algorithm for short-term scheduling of batch operations-I. MILP formulation., Computers \& Chemical Engineering 17 (1993) 211-227.

[19] C. C. Pantelides, Unified frameworks for optimal process planning and scheduling, in: D. W. T. Rippin, J. Hale (Eds.), Proceedings of the Second Conference on Foundations of Computer-Aided Operataons, CACHE Publications, 1994, pp. 253-274.

[20] L. Parshall, K. Gurney, S. A. Hammer, D. Mendoza, Y. Zhou, S. Geethakumar, Modeling energy consumption and $\mathrm{CO} 2$ emissions at the urban scale: 
Methodological challenges and insights from the United States, Energy Policy 38 (9) (2009) 4765-4782.

[21] DECC (Dept Of Energy And Climate Change), Total final energy consumption at sub-national level (2008).

URL http://www.decc.gov.uk/en/content/cms/statistics/ regional/total_final/total_final.aspx

[22] B. Boardman, S. Darby, G. Killip, M. Hinnells, C. Jardine, J. Palmer, G. Sinden, 40\% House (2005).

URL http://www.eci.ox.ac.uk/research/energy/40house.php

[23] BERR, Digest of UK Energy Statistics (2008).

URL/http://www.berr.gov.uk/energy/statistics/publications/ dukes/page45537.html

[24] J. Keirstead, N. Samsatli, N. Shah, C. Weber, D. Fisk, The Implications of CHP Planning Restrictions on the Efficiency of Urban Energy Systems, in: Proceedings of the 23rd International Conference on Efficiency, Cost, Optimization, Simulation and Environmental Impact of Energy Systems, Lausanne, 2010, pp. 1-8.

[25] T. Hartford, A marginal victory for the well-meaning environmentalist, The Financial Times.

URL http://www.ft.com/cms/s/2/b291a87c-0f9c-11df-b10f-00144feabdc0. html

[26] Vokèra, Vokèra Linea HE combi boiler (2010).

URL http://www.vokera.co.uk/home-owners/condensing-boiler/ combi-boiler/linea-he

[27] Cogen3, Available Cogeneration Technologies in Europe: Part II Technologies and Products (2003).

URL http://cogen3.net/techreportform.html 
[28] PowerTechnology.com, Avedore Multi-Fuel Power Plant, Denmark (2010).

URL http://www. power-technology.com/projects/avedore/

[29] CHPA, Fact Sheet: CHP Scales and Startup (2007).

URL http://www.chpa.co.uk/about_chp/fact_sheets/

FactSheet-CHPScales $\ \&$ Startup.pdf

[30] Ofgem, Electricity Distribution Cost Review 2005-2006 (2007).

URL http://www.ofgem.gov.uk/Networks/ElecDist/PriceCntrls/

CostRep/Documents1/16839-1807.pdf

[31] Ofgem, Gas Distribution Price Control Review: Final Proposals (2008).

URL http://www. of gem.gov.uk/Networks/GasDistr/GDPCR7-13/

Documents1/finalproposals.pdf

[32] National Grid, Seasonal and Annual Data (2009).

URL http://www.nationalgrid.com/uk/Gas/Data/misc/

[33] CTR, The Main District Heating Network in Copenhagen (2004).

URL

http://www.ctr.dk/Images/Publikationer/

Themaindistrictheatingnetworkincph-UK.pdf

[34] BERR, Quarterly Energy Prices (2009).

URL http://www.berr.gov.uk/energy/statistics/publications/ prices/index.html

[35] P. Olsen, H. Lambertsen, R. Hummelshoj, B. Bohn, C. Christiansen, S. Svendsen, C. Larsen, J. Worm, A new low-temperature district heating system for low-energy buildings, in: Proceedings of the 11th International Symposium on District Heating and Cooling, 2008, pp. 1-8. 
Table 1: Technology data. Data from [26, 27, 28, 29] and author estimates. Note that the boiler efficiency represents a modern condensing boiler, as opposed to the $70 \%$ efficient unit used to estimate heating demands from current gas demands. Technology capital costs were discounted at $6 \%$ over 15 years for the CHP units, and $10 \%$ over 10 years for the domestic technologies.

\begin{tabular}{|c|c|c|}
\hline Parameter & & Value \\
\hline \multicolumn{3}{|l|}{ 1. Boiler } \\
\hline \multicolumn{2}{|l|}{ Capital cost } & $£ 500$ \\
\hline \multicolumn{2}{|l|}{ Maintenance cost } & $£ 50 / \mathrm{y}$ \\
\hline \multicolumn{2}{|l|}{ Max capacity } & $15 \mathrm{~kW}$ \\
\hline \multicolumn{2}{|l|}{ Efficiency } & $90 \%$ \\
\hline \multicolumn{3}{|l|}{ 2. Heat exchanger } \\
\hline \multicolumn{2}{|l|}{ Capital cost } & $£ 250$ \\
\hline \multicolumn{2}{|l|}{ Maintenance cost } & $£ 50 / \mathrm{y}$ \\
\hline \multicolumn{2}{|l|}{ Max capacity } & $15 \mathrm{~kW}$ \\
\hline \multicolumn{2}{|l|}{ Efficiency } & $99 \%$ \\
\hline \multicolumn{3}{|c|}{ 3. Gas CHP (small) } \\
\hline \multicolumn{2}{|l|}{ Capital cost } & $£ 1$ million \\
\hline \multicolumn{2}{|l|}{ Maintenance cost } & $£ 35000 / y$ \\
\hline \multicolumn{2}{|l|}{ Max capacity } & $1 \mathrm{MW}_{e}$ \\
\hline \multicolumn{2}{|l|}{ Efficiency (total) } & $70 \%$ \\
\hline \multicolumn{2}{|l|}{ Efficiency (elec) } & $30 \%$ \\
\hline \multicolumn{3}{|c|}{ 4. Gas CHP (medium) } \\
\hline \multicolumn{2}{|l|}{ Capital cost } & $£ 12$ million \\
\hline \multicolumn{2}{|l|}{ Maintenance cost } & $£ 390000 / y$ \\
\hline \multicolumn{2}{|l|}{ Max capacity } & $10 \mathrm{MW}_{e}$ \\
\hline \multicolumn{2}{|l|}{ Efficiency (total) } & $80 \%$ \\
\hline \multicolumn{2}{|l|}{ Efficiency (elec) } & $40 \%$ \\
\hline \multicolumn{3}{|c|}{ 5. Gas CHP (large) } \\
\hline \multicolumn{2}{|l|}{ Capital cost } & $£ 85$ million \\
\hline \multicolumn{2}{|l|}{ Maintenance cost } & $£ 2.6$ million/y \\
\hline Max capacity & & $50 \mathrm{MW}_{e}$ \\
\hline Efficiency (total) & & $85 \%$ \\
\hline Efficiency (elec) & & $43 \%$ \\
\hline
\end{tabular}


Table 2: Other model data. Data from $[30,31,32,33,34,35]$ and author estimates.

\begin{tabular}{lr}
\hline Parameter & Value \\
\hline Electricity network & $0.03 \%$ per km \\
Losses & $£ 100000$ per km \\
Capital cost & $£ 3000$ per km-year \\
Maintenance cost & \\
Gas network & \\
Losses & $£ 300000$ per km \\
Capital cost & $£ 9000$ per km-year \\
Maintenance cost & \\
District heat network & \\
Losses & \\
Capital cost & \\
Maintenance cost & \\
Resource costs & $£ 1000000$ per km \\
Electricity & $£ 30000$ per km-year \\
\hline Gas & $£ 26.9 / \mathrm{MWh}$ \\
\hline
\end{tabular}


Table 3: Summary of technology combinations for the $8 \times 8$ city scenarios. $\mathrm{CF}=$ capacity factor, i.e. average operating rate as $\%$ of maximum operating capacity.

\begin{tabular}{|c|c|c|c|}
\hline Scenario & Technology & Number & $\mathrm{CF}$ \\
\hline Boilers & Gas boiler & 22070 & 58 \\
\hline \multirow[t]{3}{*}{ Small CHP } & CHP units & 26 & 43 \\
\hline & Heat exchanger & 17240 & 57 \\
\hline & Gas boiler & 4830 & 63 \\
\hline \multirow[t]{3}{*}{ Med CHP } & CHP units & 3 & 58 \\
\hline & Heat exchanger & 16480 & 58 \\
\hline & Gas boiler & 5590 & 60 \\
\hline \multirow[t]{3}{*}{ Large CHP } & CHP units & 1 & 30 \\
\hline & Heat exchanger & 15010 & 58 \\
\hline & Gas boiler & 7060 & 58 \\
\hline \multirow[t]{5}{*}{ All techs } & Small CHP & 8 & 45 \\
\hline & Med CHP & 2 & 50 \\
\hline & Large CHP & - & - \\
\hline & Heat exchanger & 16260 & 58 \\
\hline & Gas boiler & 5810 & 58 \\
\hline
\end{tabular}




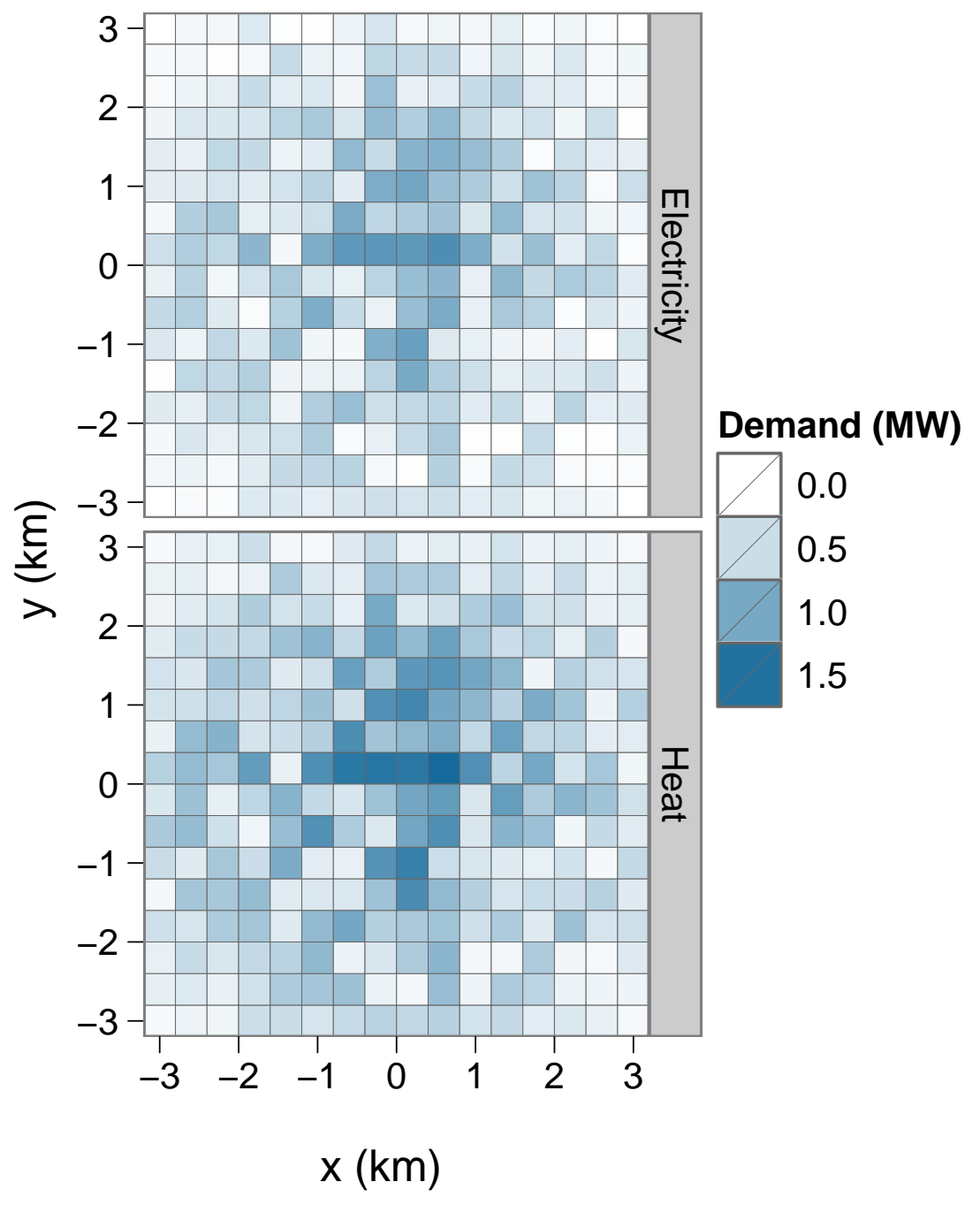

Figure 1: An indicative pattern of resource demands for a $16 \times 16$ grid city. 


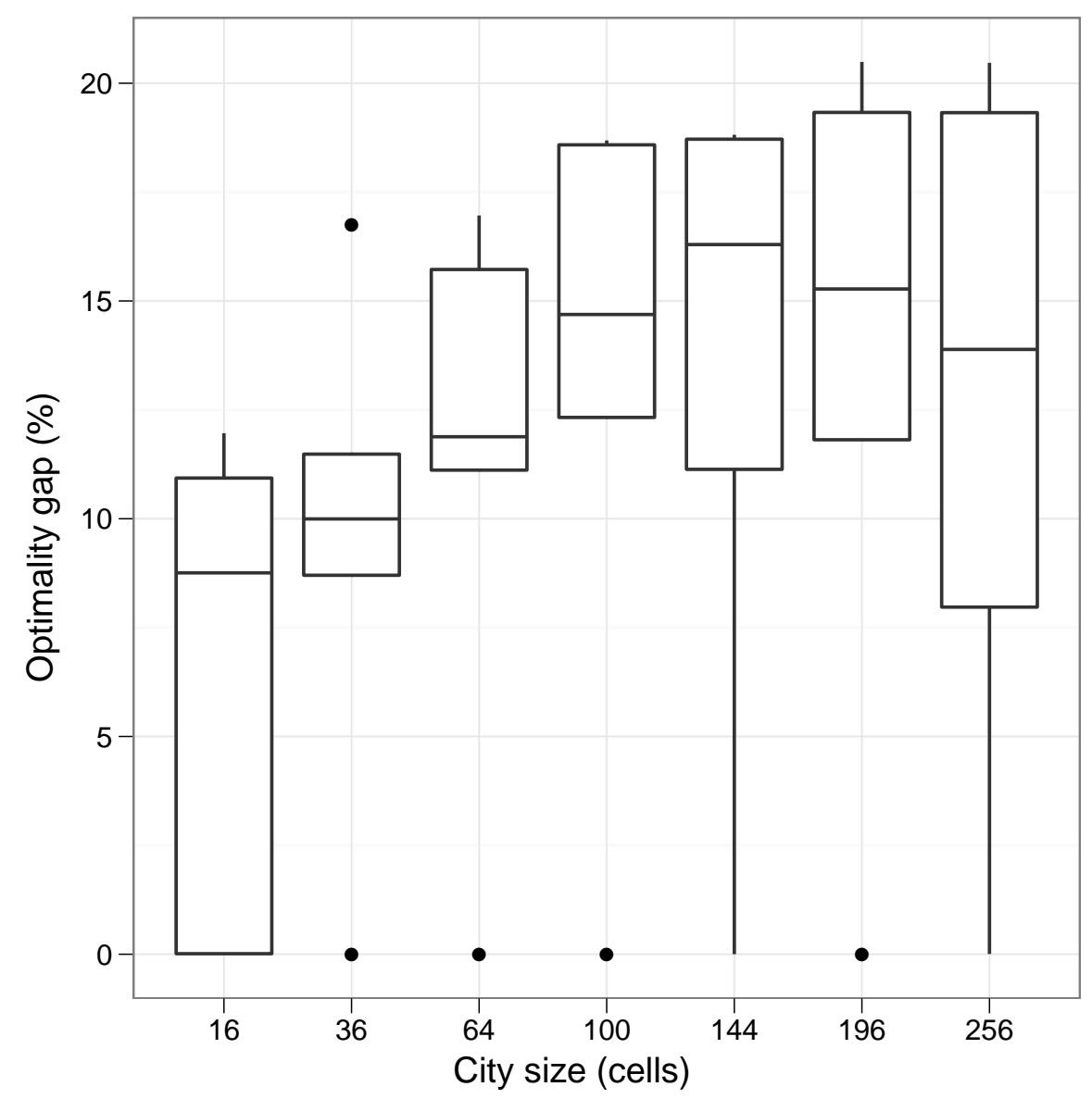

Figure 2: Summary of model solution quality. The optimality gap measures the difference in objective function value between the integer solution and the fully-relaxed LP solution. A lower value represents a better solution. 


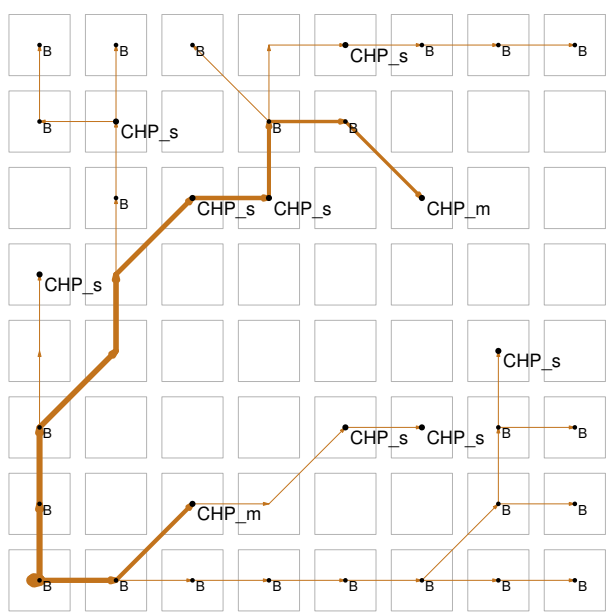

(a) Gas

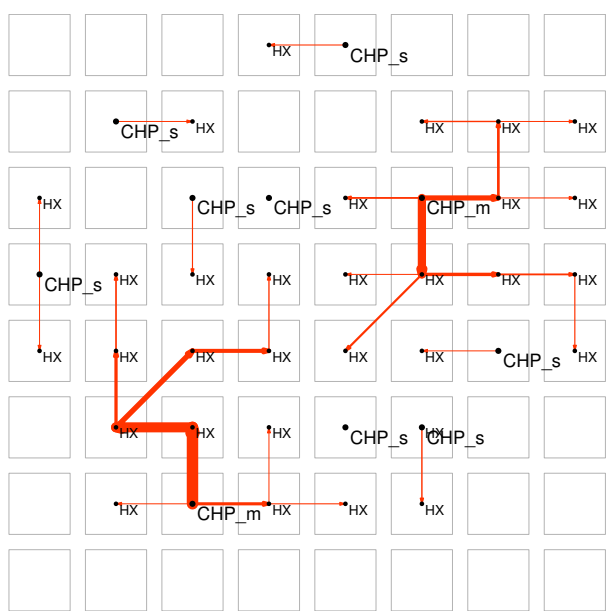

(b) District heat

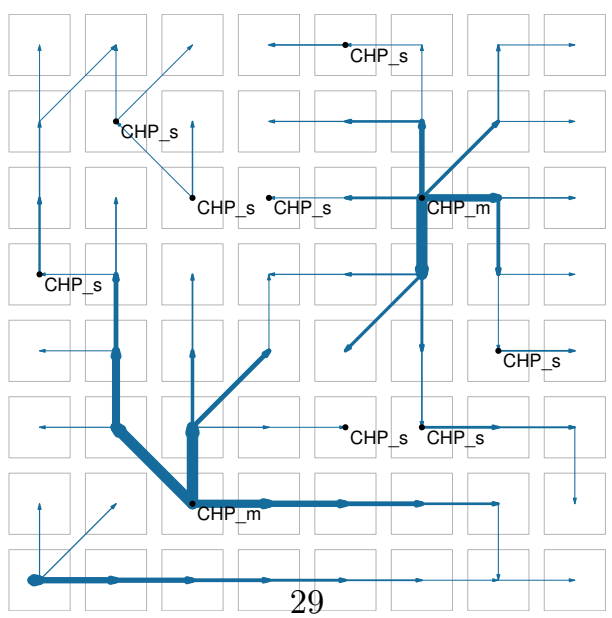

(c) Electricity

Figure 3: Resource distribution networks and technology locations for the "all technologies" $8 \times 8$ city scenario. $\mathrm{B}=$ boiler, $\mathrm{HX}=$ heat exchanger, $\mathrm{CHP}_{s}=1 \mathrm{MW} \mathrm{CHP}, \mathrm{CHP}_{m}=10$ MW CHP. 


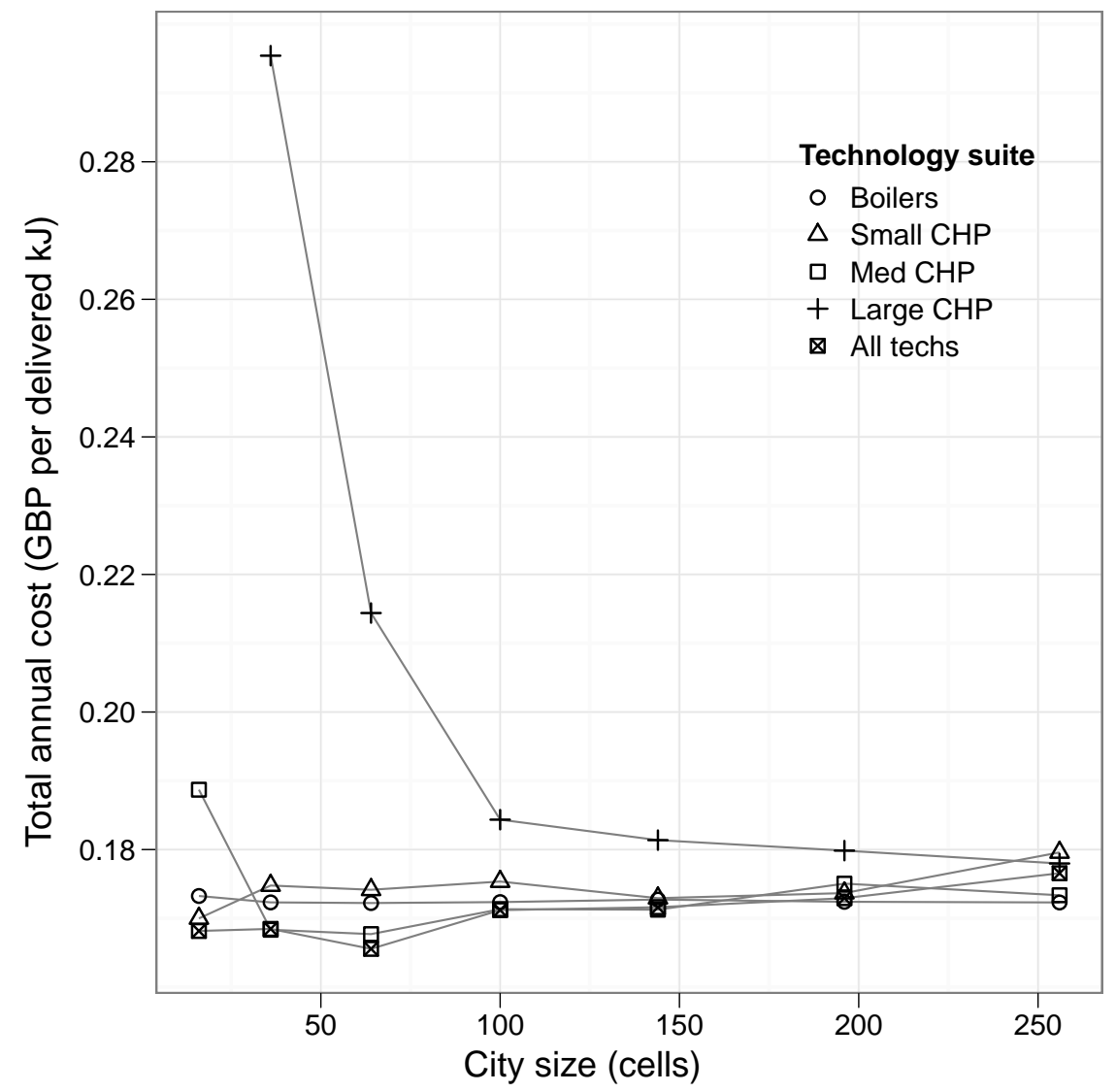

Figure 4: Cost efficiency of energy system scenarios. Costs represent annualised capital costs, maintenance costs, and fuel costs. 


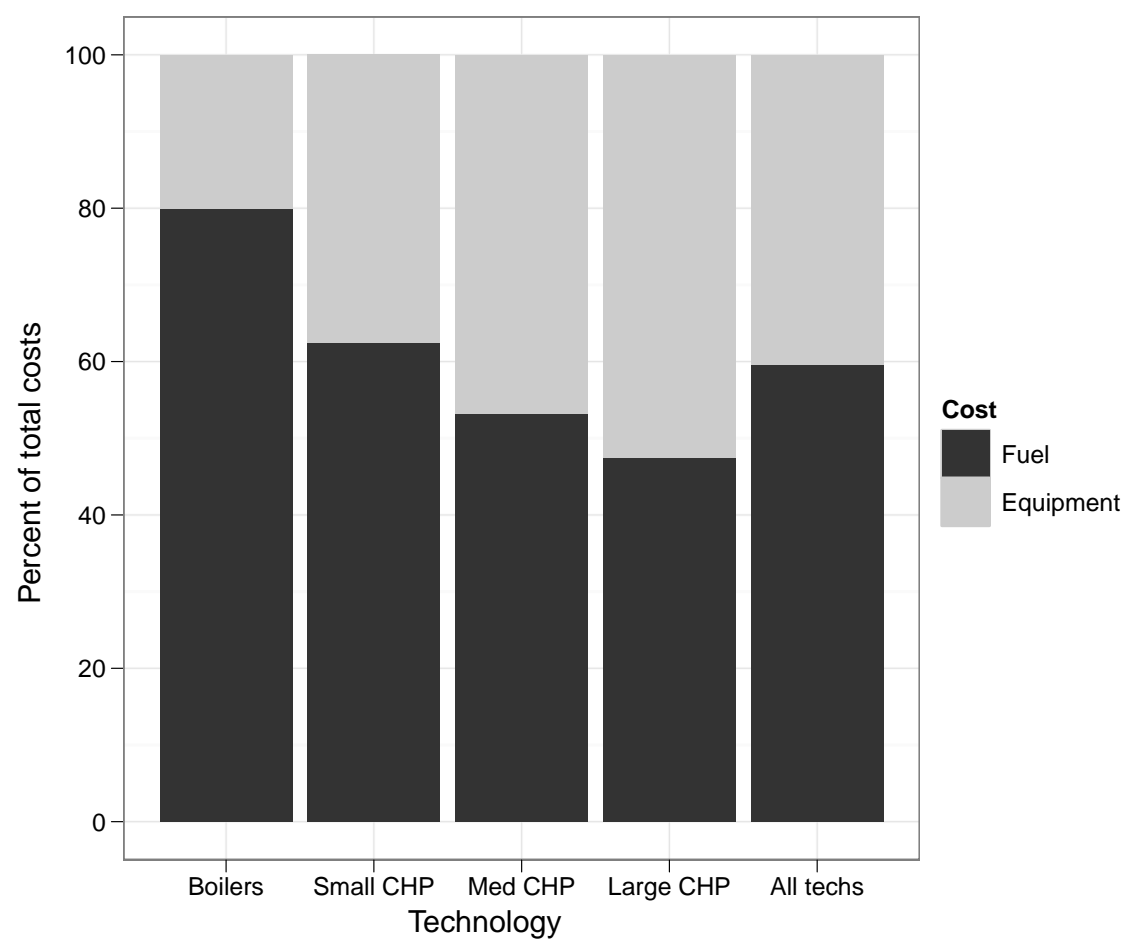

Figure 5: Constituents of total system cost for each technology scenario. 


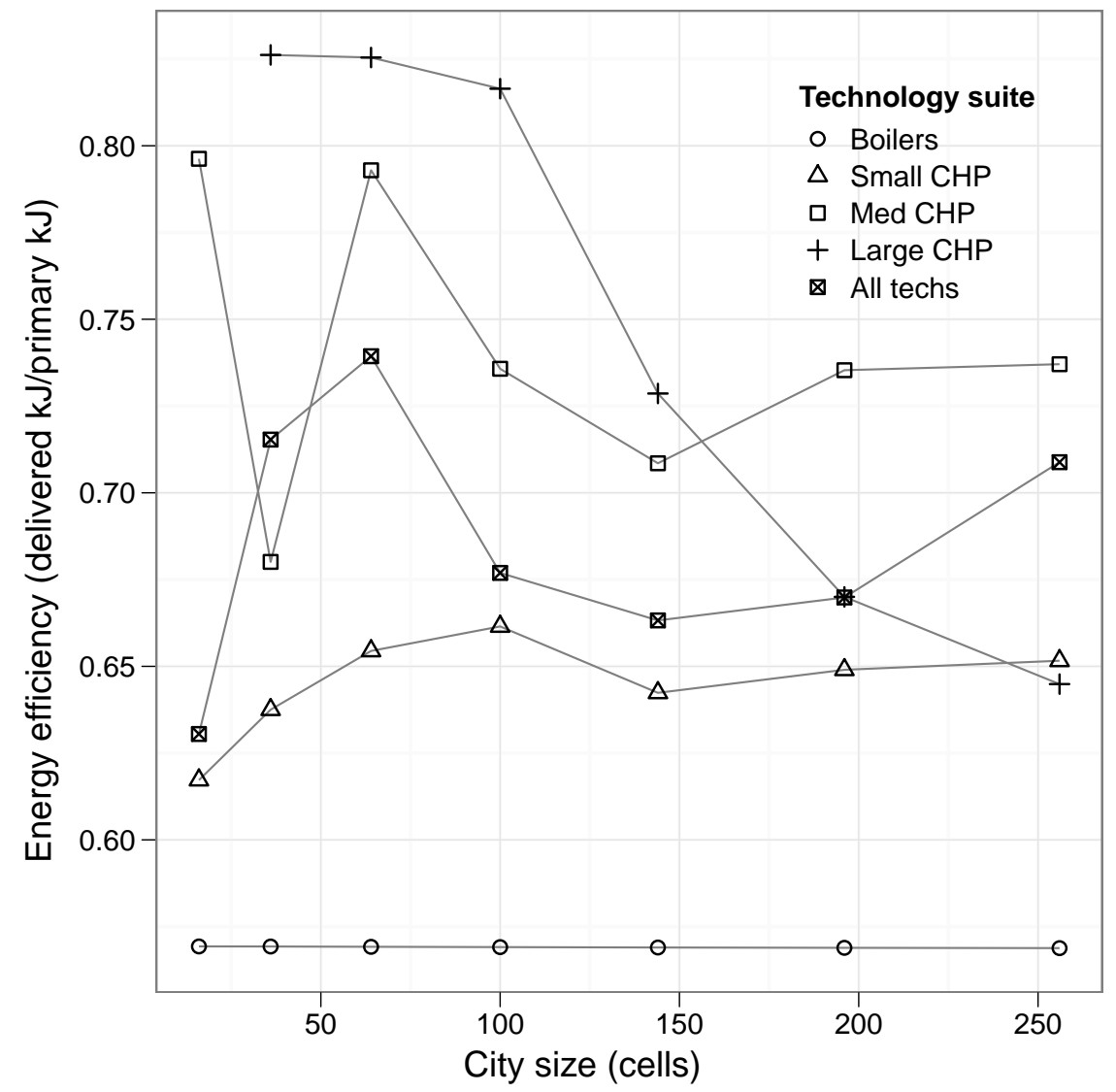

Figure 6: Energy efficiency of each scenario, as measured by delivered energy consumption per unit of primary energy (i.e. including transmission and distribution losses) 


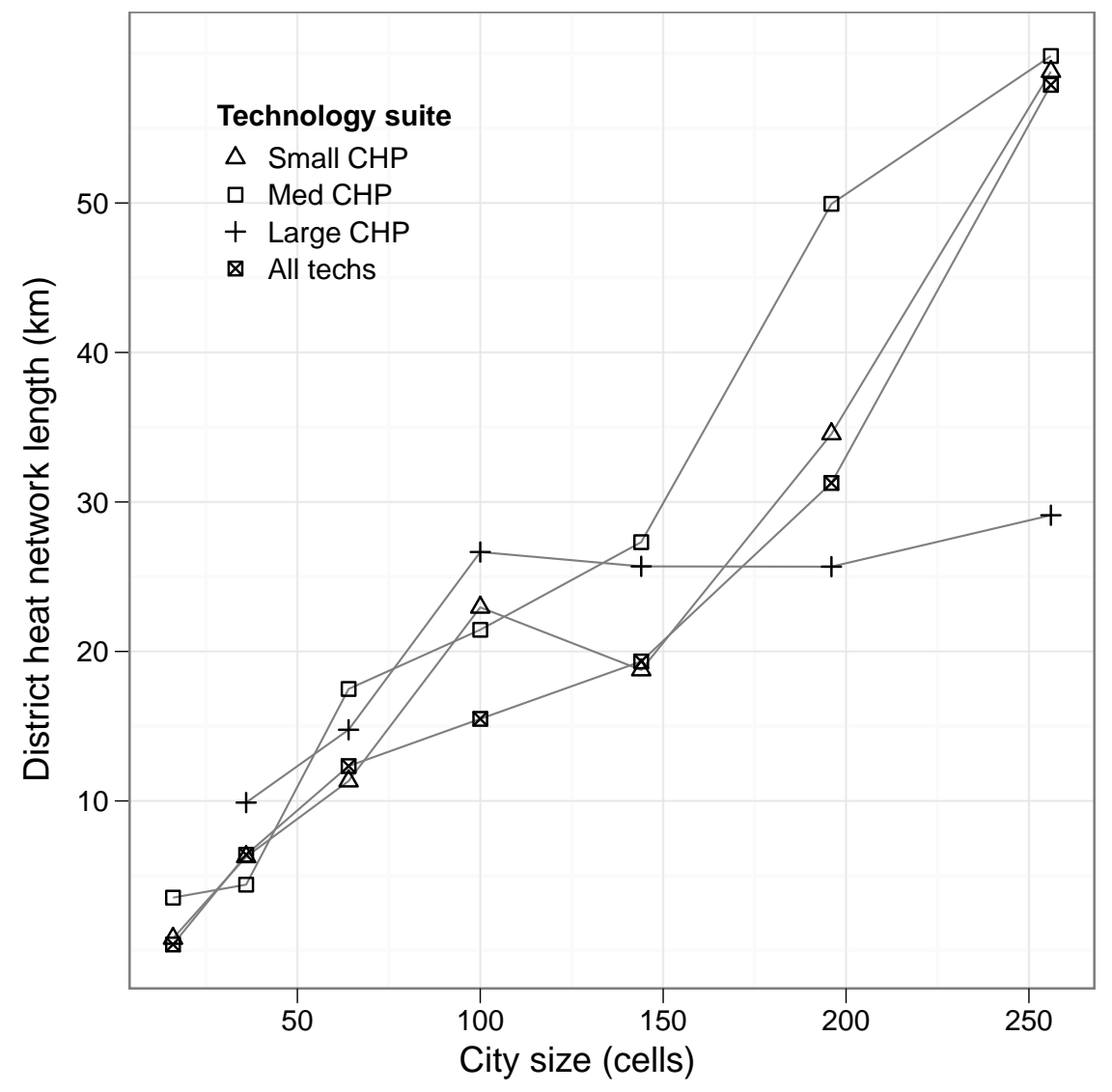

Figure 7: Length of the district heat network for each CHP scenario. 


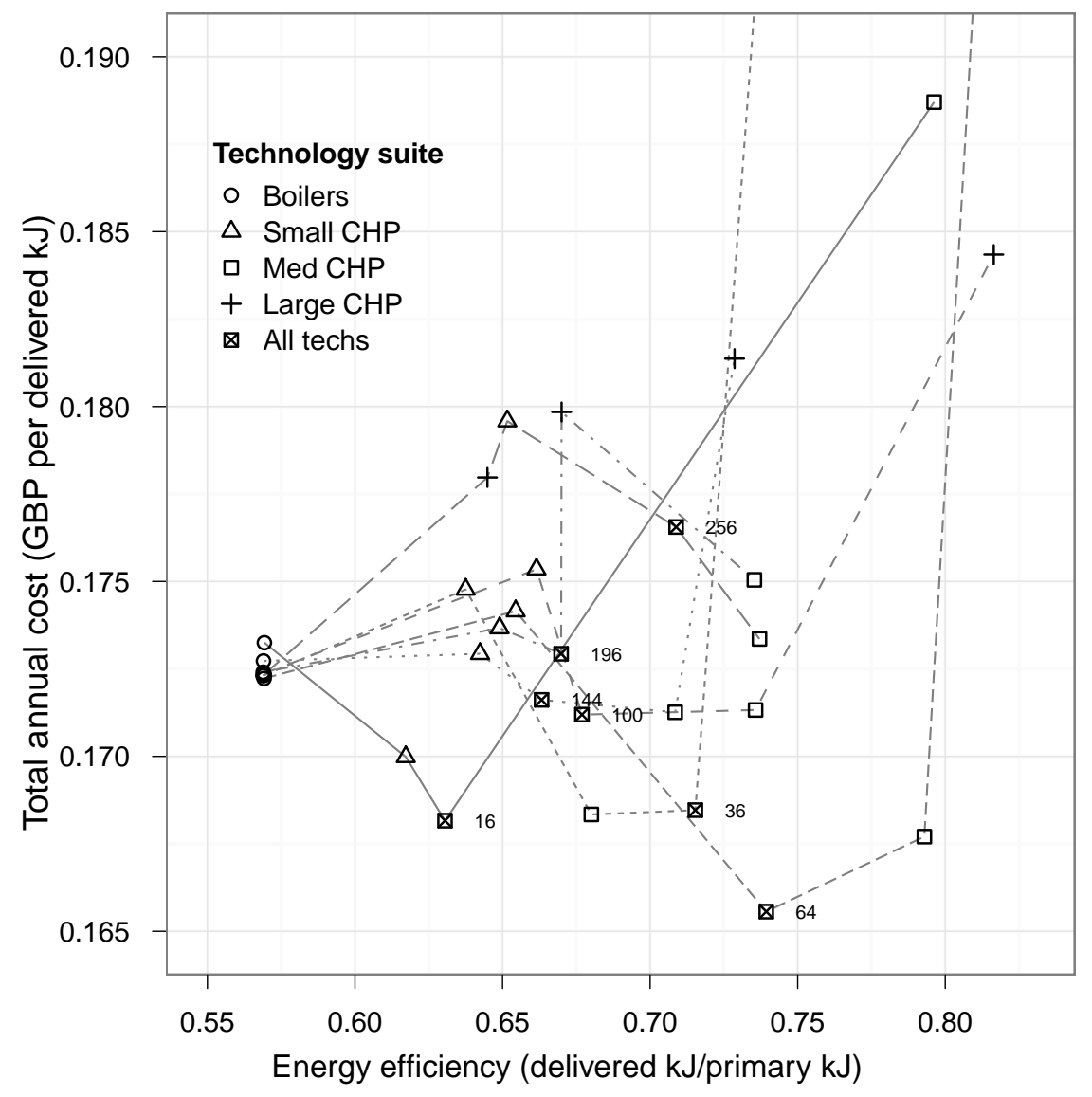

Figure 8: Summary of cost and energy-efficiency performance. Line types and numbers labelling the "all technology" data points represent city size (cells). The y-axis has been limited to exclude the $6 \times 6$ and $8 \times 8$ large CHP scenarios as they have much higher costs (see Figure 4 ). 